\title{
Correlation Coefficient Analysis of Small and Micro Businesses Database Marketing Input and Performance
}

\author{
Xuan Wang ${ }^{1, ~ a, ~ Z h o n g ~} \mathrm{Wu}^{2, \mathrm{~b}}$ \\ ${ }^{1}$ School of Business Wuhan Huaxia University of Technology, Wuhan 430223, China; \\ ${ }^{2}$ School of Physical Education, Wuhan Business University, Wuhan 430056, China. \\ acharles.fr@163.com, ${ }^{\text {b } 7849800 @ q q . c o m ~}$
}

\begin{abstract}
When small and micro businesses expand the market and make a strategic decision, do research on an internal connection between database marketing input and company's performance. When small and micro businesses carry out these, the whole performance is the fundamental starting point, small and micro businesses make the correct formulation, supervision, evaluation and optimization of database marketing input plans and program. In this paper the typical "new three board" small and micro businesses are selected, and fully analyze some quantitative demonstration analysis. The research also found a positive correlation between the whole performance of small and micro businesses and database marketing input. It proposes the corresponding rationalization proposal in base of the important parts of database marketing input activities to realize the whole enterprise performance maximization.
\end{abstract}

Keywords: Small and micro businesses; Database marketing; Performance; Correlation coefficient analysis

\section{小微企业数据营销投入与绩效相关性分析}

\author{
王旋 $1^{1} ，$ 吴钟 $2^{2}$
}

(1.武汉华夏理工学院商学院 湖北 武汉 430223；2.武汉商学院体育学院 湖北 武汉 430056)

摘要: 小微企业在做市场开拓和战略决策时, 要深入研究数据营销投入同企业绩效的内在联系, 并在具体执行过程中, 要以整体绩效作为根本出发点, 正确制定、监督、考核及优化数据营销计划和方案。本文选取具有代表性的新三板小微企业 做详尽的定量实证分析, 结果显示数据营销投入与小微企业整体绩效确实存在正相关属性, 在此基础上对数据营销活动的重 点管理环节提出相应合理化建议, 以实现企业整体绩效最大化。

关键词: 小微企业; 数据营销; 绩效; 相关性分析

中图分类号: C931 文献标志码: A

引言

科技的进步与互联网的普及催生了 “大数据” 的产生, 如今我们正在步入 “大数据时代”。大数据也 正越来越多的被应用到商业中去, 关于大数据与商业结合的成功案例不胜枚举, 然而如何将大数据应用于 小微企业的营销中去, 还是一个全新的课题。数据营销 (Database Marketing, DM) 作为一种新型的市场营 销推广手段, 结合了 IT、Database 与 Internet 技术特点, 正逐渐被市场和企业所接受, 具有广阔的市场 发展前景。

近几年, 数据营销的研究甚器尘上, 关于数据营销的文献开始有所增加。冯芷艳等认为目前国内外大 数据的研究多限于科学层面, 因此提出了大数据背景下商务管理的相关课题, 突出描述了三个视角, 即社 会化的价值创造、网络化的企业运作、实时化的市场洞察。同时指出在消费者异质化的今天大数据营销能 
够促进顾客洞察的实时化和精准化，有助于企业确立适当的市场营销策略。黄升民、刘珊认为大数据使得 营销的科学性变为可能, 全媒体和大数据使得低成本的普查成为可能, 需求被合理推测和满足, 无论是信 息还是商品服务的提供都更应趋向于定制。傅琳雅等认为大数据营销将成为未来的营销发展趋势。在大数 据时代, 消费者的信息扩大到行为层面, 在移动终端的盛行之下, 营销应该转变方法, 实现与消费者的真 实对话和良性互动。

数据营销的优势明显，但在我国发展时日尚短，企业对其仍存在较大顾虑，主要原因还是国内企业在 现阶段无法充分认识数据营销, 不敢过多投入。因此, 如何充分认识、利用好数据营销使其为企业绩效增 幅助力必将成为企业界甚至是社会各界下一阶段要共同深入探讨的重要课题。本文基于此拟构建数据营销 同企业绩效的相互关系模型, 通过数据处理分析揭示数据营销对小微企业整体绩效的实质影响, 并选取具 有代表性的小微企业进行实证研究, 对提出的假设进行相关论证, 最后提出一些有利于小微企业通过数据 营销活动实现企业绩效增幅的可行性建议。

\section{1 小微企业绩效评价指标体系建立}

数据营销的效用机制是通过对经多种渠道收集的广大消费者群体数据信息进行科学处理分析，通过数 据结果对消费者购买产品的倾向性作出预测, 从而对这些消费者倾向性的产品制作营销信息, 进行精准营 销; 同时建立消费者数据库，不断完善消费者信息，信息越全面越能给每位顾客量身定做个性化营销设计 和服务支持，从而实现 “一对一”的顾客关系管理。

通过对小微企业绩效评价内容进行全面分析，选取企业发展能力、财务效益、企业资产运营状况及偿 债能力四个指标作为小微企业绩效评价的一级指标, 在此基础上进一步细化为二级指标 ( 含基本指标、修 正指标及评议指标），从而构建小微企业绩效评价体系。选取指标的过程工作量大、难度高, 尤其是数据 搜集渠道有限, 导致很多修正指标数据无法搜集, 其次评议指标的计算过程较繁琐, 且人为因素影响较大。 鉴于此，本文构建绩效评价体系的二级指标只选取 8 个基本指标，见表 1 小微企业绩效评价体系。

表 1 小微企业绩效评价体系

\begin{tabular}{cccc}
\hline 评价内容 & 指标名称 & 计算公式 & 性质 \\
\hline \multirow{2}{*}{ 资产运营状况 } & 总资产周转率 & 主营业务收入净额/平均资产总额 & 正指标 \\
& 流动资产周转率 & 主营业务收入净额/平均流动资产总额 & 正指标 \\
\hline & 资本积累率 & 本年所有者权益增长额/年初所有者权益 & 正指标 \\
& 营业增长率 & 本年主营业务增长额/ & \\
& 已获利息倍数 & 上年主营业务收入总额 & 正指标 \\
& 资产负债率 & 税前利润总额/利息支出 & 适度指标 \\
\hline \multirow{2}{*}{ 偿债能力状况 } & 净资产收益率 & 债总额/资产总额 & 适度指标 \\
\hline 财务收益状况 & 总资产报酬率 & 税前利润总额/平均资产总额 & 正指标 \\
\hline
\end{tabular}

\section{2 小微企业数据营销投入与绩效回归分析}

\section{1 假设的提出}

市场竞争日趋激烈，小微企业要想在市场中赢得一席之地就必须要在不断推出有竞争力的产品的基础 
上加大、改进及完善相关营销工作，充分发挥营销效用有效提升市场份额。企业在进行营销活动时，除了 要注意方式的多样性、有效性之外, 还要综合考虑营销活动的费用情况, 尤其是营销的投入产出比, 要不 断优化营销方案, 以期取得最优营销效果, 以最低的营销成本换来最大的企业绩效增幅。为此本文做出以 下假设：

H1：小微企业数据营销投入成本与企业绩效存在显著的正相关属性。

\section{2 样本的选取}

本文对于研究样本的选取进行了重点分析, 首先排除消费弹性较大的企业, 如奢侈品企业等, 接着重 点考虑与人们日常生活紧密关联的企业, 尤其是小微企业, 在此范围内最终选择营销活动具有数据营销特 点的五家新三板小微企业作为样本，具体为金刚游戏、腾龙电子、双杰电气、淘礼网、汉唐自远，以上 5 家企业均为数据营销企业, 利用常见的数据营销手段进行营销决策和分析, 本文的数据均来源于中国经济 网站。

\section{3 小微企业绩效评价指标权重的确定}

各个指标在企业绩效评价中所占重要程度不同，基于客观性及科学性的原则，本文选取”拉开档次法” 对 8 个二级绩效评价指标赋权, 通过对 5 家样本小微企业的数据进行处理分析得出 8 个二级指标权重, 具 体数值见表 2 小微企业绩效评价指标权重表。

表 2 小微企业绩效评价指标权重表

\begin{tabular}{cccc}
\hline 评价内容 & 指标名称 & 计算公式 & 指标权重 \\
\hline \multirow{2}{*}{ 资产运营状况 } & 总资产周转率 & 主营业务收入净额/平均资产总额 & 12.01 \\
& 流动资产周转率 & 主营业务收入净额/平均流动资产总额 & 14.22 \\
& 资本积累率 & 本年所有者权益增长额/年初所有者权益 & 11.72 \\
& 营业增长率 & 本年主营业务增长额/ & \\
& 上获利息倍数 & 上年主营业务收入总额 & 8.97 \\
& 资产负债率 & 税前利润总额/利息支出 & \\
\hline \multirow{2}{*}{ 偿债能力状况 } & 负债总额/资产总额 & 6.35 \\
& 净资产收益率 & 净利润/平均净资产 & 12.52 \\
\hline 财务收益状况 & 总资产报酬率 & 税前利润总额/平均资产总额 & 21.11 \\
\hline
\end{tabular}

\section{3 小微企业数据营销投入与绩效回归分析}

本文拟选用 CURVEFIT 模型来进行数据营销与企业绩效关系模型拟合。CURVEFIT 模型具有两方面的优 点: 一方面其可以检验可能存在的模型线性关联; 另一方面其还能对其他 10 个关联模型进行相关检验（包 括直线、逆、立方、指数、指数分布、增长、复合、二次、 $S(S)$ 、对数等）。CURVEFIT 模型可以从成对 测量值（根据经验存在）的排列中截取测算出相关直线函数。除此之外，其还可以针对每个相关的函数均 测算出相应的统计参数, 如 R2 及显著性等。综上所述, CURVEFIT 模型可以根据相关统计参数的情况在不 同的曲线模型之间进行比较。此外，通过不同曲线模型之间的比较还可以得出哪个函数可能比直线模型能 
够更好反映所要调查分析的两变量之间的关联。输出结果见表 3 。

表 3 模型总结和参数估计值

\begin{tabular}{|c|c|c|c|c|c|c|c|c|c|}
\hline \multirow[b]{2}{*}{ 方程 } & \multicolumn{5}{|c|}{ 模型总结 } & \multicolumn{4}{|c|}{ 参数估计值 } \\
\hline & $\mathrm{R}^{2}$ & F & $\begin{array}{l}\text { 自由 } \\
\text { 度 } 1\end{array}$ & $\begin{array}{l}\text { 自由 } \\
\text { 度2 }\end{array}$ & 显著性 & 常量 & b1 & b2 & b3 \\
\hline Linear & .304 & 1.554 & 1 & 365 & .213 & 2.329 & -.071 & & \\
\hline Logarithmic & .691 & .541 & 1 & 365 & .042 & 3.242 & .111 & & \\
\hline Inverse & .600 & .021 & 1 & 365 & .885 & 2.112 & .050 & & \\
\hline Quadratic & .626 & 4.794 & 2 & 364 & .009 & .964 & .922 & -.173 & \\
\hline Cubic & .432 & 4.039 & 3 & 363 & .008 & 2.569 & -1.042 & .579 & -.091 \\
\hline Compound & .304 & 1.537 & 1 & 365 & .216 & 3.234 & .969 & & \\
\hline Power & .759 & .512 & 1 & 365 & .014 & 3.145 & -.049 & & \\
\hline $\mathrm{S}$ & .500 & .008 & 1 & 365 & .931 & .709 & .014 & & \\
\hline Growth & .204 & 1.537 & 1 & 365 & .475 & .804 & -.032 & & \\
\hline Exponential & .004 & 1.537 & 1 & 365 & .016 & 2.234 & -.032 & & \\
\hline Logistic & .004 & 1.537 & 1 & 365 & .016 & .448 & 1.032 & & \\
\hline
\end{tabular}

依据表 3 的输出参数, 选择相适应的回归方程有一个重要的前提条件, 即变量的确定要跟回归方程的 应用目的相匹配。其中 $\mathrm{x}$ 值函数与最大值和最小值范围拟合是变量对于模型是否匹配的一个重要性提示, 如果存在数据不相同、数值指向不相同的情况，则说明该函数没能做到与数据的较好拟合，不适合采用该 函数进行预测; 即使判定存在最好的参数，但是如果测定的相应曲线完全没能反应预期的或者根据经验存 在的分布，那这些参数即使再好也是没有什么意义的。在满足以上所述条件外，还要同时满足相对简单性 及显著性两个标准。

选取满足显著性的回归方程通常分两步，第一步观察所有能使 $\mathrm{F}$ 检验结果达到显著性的曲线函数，第 二步在已选择的曲线函数范围内观察 R2 数值最高的曲线函数。满足相对简单性是选取适合的回归方程的 另一个准则, 如要选取更好的模型参数通常意味着要面临更复杂的回归方程, 且这种复杂性无法总是通过 改善较小的模型拟合度数值来达到降低的目的, 这其中一个典型例子: 一个三次回归模型同线性回归模型 的 R2 差别只有 0.001 , 但是需要增加两个变量, 计算量和复杂性大幅增加, 通常在这种情况下, 宜以相对 简单性为原则选取线性回归方程，且这样选择还可带来其他好处，其一是采用线性回归模型及线性相关模 型进行计算不会引起实质性信息丢失的情况发生，其二是线性模型对计算结果的解释要较三次模型更简 单。

由表 3 数据可知, 直线、复合、逆、增长、S ( S) 5 个标准函数不符合显著性标准，可以删除; 此外, 以最好的参数 $\mathrm{R} 2$ 为选择标准应选择幂函数, 但是在加入相关数据后幂函数呈增函数了, 这与根据实际经 验存在的分布不相符。综合考虑, 最终确定对数函数 $\mathrm{y}=3.242+0.1111 \mathrm{n}(\mathrm{x})$ 。

回归分析一般具有两方面的功能: 一方面是解释功能, 另一方面是预测功能。解释功能主要是能够说 明两个变量之间的关联方向及关联强度; 预测功能发挥的途径是通过构建回归模型, 在已知的自变量的基 础上预测未知的因变量。最终确定的对数函数 $\mathrm{y}=3.242+0.1111 \mathrm{n}(\mathrm{x})$ 经过数据结果分析, 可得出数据营销投 入与企业绩效的关联强度达到了较好的标准，满足了基本的要求。 


\section{4 结论}

营销活动与绩效是相互关联的，一方面营销可以促进销售，实现营业收入的增加，进而通过利润的提 高最终实现企业绩效的增加; 一方面企业绩效向好的方向发展又会加大营销的资源投入，包括配备更多的 营销人员、投入更多实质性的营销费用等, 这样形成一个良性闭环。企业内部称营销部为 “企业利润中心” 是对其关联性的一个佐证。且通过以上的分析得出在提升小微企业整体绩效方面, 加大数据营销活动的投 入是非常直接有效的方式。

\section{1 数据营销活动中财务管理}

一切的营销活动包括数据营销活动都必须以市场为风向标，根据市场实际情况做好营销策划，采用多 种营销形式发展新的客户群和吸引已有的客户群重复购买, 以期能在市场同类产品的竞争中占得先机。营 销活动最直接的结果就是实现产品销售量的增加, 带来销售收入的增长, 但是一定不能忽视营销成本的投 入, 如果一味不计成本投入, 只以销售量增加为唯一目标则很有可能会取得适得其反的效果。因此, 在营 销活动的策划、执行和结果分析的过程中一定要做好成本测算，做好财务管理。

小微企业一般是首先通过一款明星产品就能打开市场知名度，但是要想在残酷的市场竞争中持续发展 壮大, 就需要不断的更新已有产品, 不断开发新的产品以丰富产品类别, 这就是小微企业的多元化经营理 念。但多元化经营意味着需要更多的研发资金和流动资金, 且一款新的产品即使在市场上能被消费者接受, 也会存在一个的过程, 甚至有些新的产品在市场上不被接受即淘汰, 这样企业就有很大一部分研发资金和 流动资金被圈住甚至是面临损失。因此小微企业在采用多元化经营策略之前要做好详细的市场调查, 这样 能一定程度上规避财务风险。

\section{2 数据营销活动中风险管理}

小微企业要想持续发展壮大, 就必须不断开拓市场, 开展各种形式的营销活动成为必然, 营销费用 ( 含 营销人员的开支、运营费、广告费、数据服务费用等）在企业运营成本中所占的比例会随着市场竞争加剧 持续升高, 因此企业应从整体运营和市场开发的角度出发, 进行合理的数据营销规划。但在实际操作过程 中, 数据营销的管理仍存在较大问题, 其一目标不明确, 导致数据营销结果偏离当初的目标; 其二是数据 营销方式不合理，造成了巨大的浪费。如果数据营销管理，最终上升到企业绩效风险。

如今, 业界已经从对数据重要性的认识阶段, 发展到实践数据的必要性的战略实施阶段, 因此, 如何 保证数据的真实性、如何挖掘数据价值成为大数据应用的关键问题。本文提供 5 个方面的指导建议:

（1）以 “客户为中心”，制定前期 “数据营销战略规划”;

（2）制定全面完整的企业 “数据蓝图”;

（3）从现有数据入手，设定并完成短期和阶段性的 “数据营销战略目标”;

（4）根据业务优先级，逐步建立分析体系，循序渐进提升 “数据分析能力” ;

（5）定制可衡量的指标分析 “数据 ROI（投资回报率）”。

综上所述, 企业营销投入与绩效呈现出显著的正向相关关系，即企业营销投入大, 绩效也是越大的。而 且在复杂的市场环境中, 企业绩效除了受企业营销投入的影响, 还受到经济环境、企业营销能力等因素的 影响, 企业营销投入与营销绩效不是完全线性相关的, 一般情况而言, 它将呈现出正向的不完全相关关系。 本文的局限性主要是模型只能说明营销投入与企业绩效的相关程度, 不能说明二者的实际过程及结果, 然 
后相对于整个市场容量而言, 调查选取的样本容量比较小, 抽样本身就具有风险, 不能避免数据与市场实 际的存在偏差, 后续研究应取得更多的样本数据来进行研究。

\section{5 致谢}

本文得到了教育部人文社会科学研究青年基金项目 “基于本体的 020 服务均衡推荐方法研究”（编号: 14YJCZH165) 和武汉华夏理工学院基金项目: “互联网 + "背景下的小微型企业数据化运营研究 ( 编号: 15025) 资助。

\section{Acknowledgement}

This paper is supported by the Humanity and Social Science Youth foundation of Ministry of Education of China (No.14YJCZH165) and the Foundation of Wuhan Huaxia University of Technology (No. 15025).

\section{参考文献:}

[1] 冯芷艳, 郭迅华, 曾大军, 陈显波, 陈国青. 大数据背景下商务管理研究若干前沿课题 [J]. 管理科学学报. 2013, $16(1): 1-9$

[2］黄升民, 刘珊. “大数据” 背景下营销体系的解构与重构 [J]．现代传播(中国传媒大学学报)．2012，34 (11):13-20

[3] 傅琳雅. “互联网+文化产业” 的新业态及发展趋势 [J]．沈阳工业大学学报(社会科学版)．2016，9 (4): 306-309

[4] 马智萍. 大数据时代的中小企业营销创新方式选择. 商业经济研究，2015（4）：62-63.

[5］袁清. 大数据时代营销体系的重构与发展思考. 商业经济研究，2016,（24）：46-48.

［6］李海波. 电子商务背景下个性化精准营销策略研究. 商业经济研究, 2016,（2）：42-44.

[7] 张瑜. 旅游网络营销绩效评估研究 [D]. 延安大学, 2013.

[8］曹喜民．企业营销模式对企业绩效的影响研究 [D]．西安工业大学，2014.

[9] 陈正昌. SPSS 与统计分析 [M]. 教育科学出版社: 北京, 2015.

[10] 王益锋，曹禺. 科技型小微企业商业模式创新影响因素分析 [N]. 科技型小微企业创新发展论坛: 2013, $30(18): 13-17$

\section{References}

[1] Feng Zhi-yan, GuoXun-hua: "On the research frontiers of business management in the context of Big Data"[J].Journal of Management Sciences in China, Vol.1(2013),No.16,p.1-9 (In Chinese)

[2] Huang Sheng-min,Liu Shan: "Deconstruction and reconstruction of marketing system under the background of "Big Data"' [J]. Modern Communication (Journal of Communication University of China)d Management, Vol. 34(2012),No.11,p.13-20 (in Chinese)

[3] Fu Lin-ya: "New format and development trend of Internet plus cultural industry" [J]. Journal of Shenyang University of Technology (Social Science Edition), Vol.9 (2016), No.4, p.306-309 (In Chinese).

[4] Ma Zhi-ping: "The choice of marketing innovation of small and medium sized enterprises in the era of Big Data" [J]. Journal of Commercial Economics, Vol. 4(2016), p.62-63. (In Chinese).

[5] Yuan Qing: "The reconstruction and development of the marketing system in the era of big data" [J]. Journal of Commercial Economics, Vol. 24(2016), p.46-48. (In Chinese).

[6] LiHai-bo: "The research of personalized precision marketing strategy under the background of e-commerce" [J]. Journal of Commercial Economics, Vol. 2(2016), p.42-44. (In Chinese). 
[7] SunYu: Evaluation of Tourism Network Marketing Performance (MS., Yan'an University, China 2013), p.36-39.

[8] Cao Xi-min: Study on the Impact of Corporation Marketing Models on Corporate Performance (MS., Xi'an Technological University, China 2014), p.29-35.

[9] Chen Zheng-chang: "SPSS and Statistical Analysis" [M].Journal of Education Science, Beijing (2015), p.52-80. (In Chinese).

[10] Yuan Qing: "Study on the Influence Factors of Business Model Innovation of Small and Micro-sized Sci-tech Enterprises" [N]. Science \& Technology Progress and Policy, Vol. 30(2013), No.18, p. 13-17. (In Chinese). 